\title{
Research Report Luminometric Assay of Platelet Activation in 96-Well Microplate
}

BioTechniques 31:1174-1181 (November 2001)

\author{
B. Sun, N.N. Tandon, \\ N. Yamamoto, M. Yoshitake, \\ and J.-i. Kambayashi \\ Maryland Research Institute, \\ Rockville, MD, USA
}

\section{INTRODUCTION}

Platelets play an important role in hemostasis. Platelets have been shown to be involved in the pathogenesis of atherosclerosis and restenosis after angioplasty. They also play a vital role in myocardial infarction and stroke. It has been documented that anti-platelet agents are effective in preventing the recurrence of infarction/stroke and restenosis. While many advanced techniques exist for developing these antithrombotic drugs, the progress of developing newer anti-platelet drugs with better efficacy has been hindered by the lack of a large-scale functional screening method (4). Currently, the most commonly used method to detect platelet activation by a given agonist is aggregometry $(3,8)$. A one- to eightchannel aggregometer has been developed for the detection of platelet activation in platelet-rich plasma (PRP), washed platelets (WP), gel-filtered platelets (all by light transmission and scatter), and in whole blood (WB) (by impedance). These assays require one to assemble a cuvette with a stir bar, followed by the pre-warming of the assembled apparatus and sample at $37^{\circ} \mathrm{C}$. Drawbacks to using this method to detect aggregation are that a large volume of sample (at least $300 \mu \mathrm{L}$ ) and a time frame of 5-10 min are required to complete each assay. Flow cytometry has also been used to detect platelet activation by detecting the expression of surface markers such as P-selectin, integrin $\alpha$ II $\beta$ III, and microparticles $(7,11)$. However, this method is quite tedious because it requires the labeling, fixing, and washing of the first and second antibodies. In addition to these tech- niques, platelet attachment to precoated solid surface (collagen and laminin) has also been used to measure platelet adhesion via standard/fluorescence microscopy or protein/enzyme quantification (flow or static adhesion assay) $(1,13)$. Specific instruments have been developed and used for diagnostic purposes and clinical trials. The PFA$100^{\mathrm{TM}}$, developed by Dade International Incorporated (Miami, FL, USA), was used as an introductory quantitative measurement of platelet function in WB under high-shear conditions. The Clot Signature Analyzer ${ }^{\circledR}$ was developed by the Xylum Corporation (Scarsdale, NY, USA) for the evaluation of global hemostasis and coagulation functions in WB (in vitro). All of the techniques mentioned above are either time consuming, expensive, or unsuitable for multiple tests simultaneously. Although the 96-well microplate has been used previously to detect platelet activation $(2,5,10)$ and coagulation (14) in PRP by monitoring light absorption (OD), the window of detection is small. Therefore, its sensitivity is limited.

Platelets contain two kinds of granules, dense and $\alpha$ granules. The dense granules contain serotonin, ADP, ATP, PF4, etc. The $\alpha$ granules, on the other hand, contain large molecules of protein (9). Upon platelet activation by various agonists, the platelets release the content of their granules. The detection of released ATP by luminescence, in the presence of luciferase/luciferin using an aggrego-luminometer, has been used to measure platelet degranulation. We have applied the same principal (i.e., detection of released ATP by luminometry) to develop this assay for measuring platelet activation using a 96-well microplate. 


\section{MATERIALS AND METHODS}

\section{Preparation of PRP}

Venous blood was drawn, following in-house safety committee-approved procedure, from healthy volunteers who had not taken any medication in the past 10 days. All volunteers were required to sign a consent form for venipuncture. Blood was collected by a two-syringe technique, with the first 2 $\mathrm{mL}$ of blood discarded, using a 19gauge butterfly needle (12) and mixed with $3.8 \%$ sodium citrate $(9: 1$ by volume) or with recombinant human hirudin $(100 \mathrm{U} / \mathrm{mL})$. PRP was prepared by centrifuging the blood in $15-\mathrm{mL}$ conical tubes at $150 \times g$ for $20 \mathrm{~min}$ at room temperature (model TJ-6 benchtop centrifuge; Beckman Instruments, Fullerton, CA, USA). The straw-colored upper phase (PRP) was collected without disturbing the interphase (buffy coat) and the lower phase (packed red cells).

\section{Preparation of WP}

Citric acid (1 M) was added to PRP at $10 \mu \mathrm{L} / \mathrm{mL}$ and gently mixed. Platelets were then pelleted at $1000 \times g$ for 10 $\mathrm{min}$. After removing the plasma by vacuum, the remaining plasma on the tube wall was carefully wiped off using a cotton swab. The pelleted platelets were then resuspended in Tyrode's HEPES buffer $(136.7 \mathrm{mM} \mathrm{NaCl}, 5.5 \mathrm{mM}$ dextrose, $2.6 \mathrm{mM} \mathrm{KCl}, 13.8 \mathrm{mM} \mathrm{NaHCO}_{3}$, $1 \mathrm{mM} \mathrm{MgCl} 2,0.36 \mathrm{mM} \mathrm{NaH}_{2} \mathrm{PO}_{4}$, and $10 \mathrm{mM}$ HEPES, $\mathrm{pH}$ 7.4) and $20 \mu \mathrm{g} / \mathrm{mL}$ heparin (Elkins-Sinn, Cherry Hill, NJ, USA). The number of platelets was counted using a Coulter ${ }^{\circledR} \mathrm{ZM}$ cell counter (Coulter, Miami, FL, USA), and the platelet concentration was adjusted to the desired level for experimental needs.

\section{6-Well-Based Platelet Activation Assay}

PRP, WP, or WB in a volume of $80-180 \mu \mathrm{L}$ was added to each well of an all-white 96-well microplate (Packard Instrument, Meriden, CT, USA). In the case of testing the inhibitory effects, inhibitors $(1 \mu \mathrm{L})$ were added to with the platelets and incubated at $37^{\circ} \mathrm{C}$ for $10 \mathrm{~min}$. The platelets were then stimu- lated with $20 \mu \mathrm{L}$ of the appropriate agonists [collagen from Chrono-Log (Haverton, PA, USA); U46619 from Biomol (Plymouth Meeting, PA, USA); and thrombin and 2-methylthio-ADP (2-MeS-ADP) from Sigma (St. Louis, MO, USA)]. 2-MeS-ADP was purified by reverse-phase HPLC to remove any ATP contamination. Following stimulation, the plate was placed on a Delfia ${ }^{\circledR}$ plateshake (model 1296-004; Applied Biosystems, Turku, Finland) to be shaken on setting no. 6 for $1-5 \mathrm{~min}$ at room temperature. Lastly, $50 \mu \mathrm{L}$ Chrono-lume (Chrono-Log) were injected into each well. Luminescence in arbitrary units was detected for $1 \mathrm{~s}$, following a 1-s delay, using a Mediators $\mathrm{PhL}$ luminoplate reader (ImmTech, Rockville, MD, USA).

\section{6-Well-Based Clotting Assay}

Initially, $50 \mu \mathrm{L}$ citrated PRP were added to each well. Heparin $(1 \mu \mathrm{L})$ at different concentrations was added to the PRP. Then, $20 \mathrm{mM} \mathrm{CaCl} 2$ (final concentration) in $50 \mu \mathrm{L}$ saline were added. The plate was then placed in a $37^{\circ} \mathrm{C}$ incubator for $20 \mathrm{~min}$. After equilibration at room temperature for $5 \mathrm{~min}$, $50 \mu \mathrm{L}$ Chrono-lume were injected, and

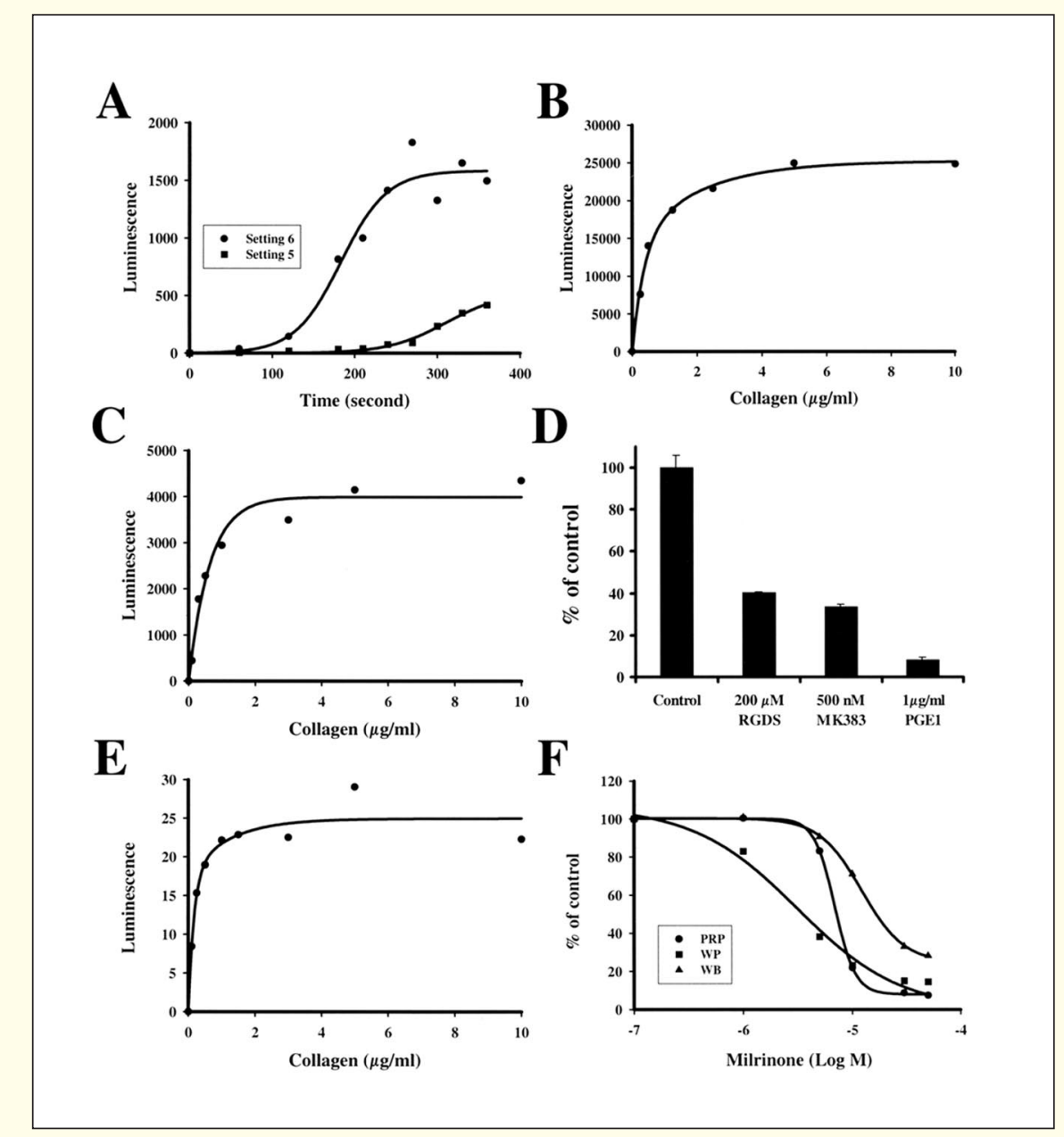

Figure 1. Collagen-induced platelet activation as determined by the 96-well-based luminometry. (A) Time course for collagen $(1 \mu \mathrm{g} / \mathrm{mL}$ )-induced platelet activation in PRP at two shaking settings. (B, C, and E) Representatives of dose-dependent effects of collagen on platelet activation in WP, PRP, and WB, respectively. The platelet count was the same for WP and PRP $\left(4 \times 10^{8} / \mathrm{mL}\right)$. Each data point represents the mean of duplicate assays. Similar data were obtained from at least two other separate experiments. Luminescence data are in arbitrary units. (D) Inhibitory effect of RGDS, MK383, and PGE $_{1}$ on collagen $(1 \mu \mathrm{g} / \mathrm{mL})$-induced platelet activation in PRP. Data are expressed as the percentage of the controls; $n=5$. (F) Concentration-dependent inhibition of collagen $(1 \mu \mathrm{g} / \mathrm{mL})$-induced platelet activation by milrinone in PRP, WP, and WB. Data are the mean of two separate experiments with duplicate assays and expressed as the percentage of the controls (without milrinone). 
the luminescence in arbitrary units was detected as above.

\section{Data Processing}

Each data point was assayed in duplicates. Where applicable, the data were expressed as the mean \pm SD of the mean. Curve fit was performed using SigmaPlot (SPSS, Richmond, CA, USA).

\section{RESULTS}

The activation of platelets in a 96well microplate as described above was dependent on time, platelet concentration, and shaking intensity. Two settings for shaking the microplate are shown in Figure 1A. There are 10 settings on the shaker, with shaking intensities from 100 to $1350 /$ min. Setting no. 6 was most suitable to the activation of platelets in PRP by collagen (1 $\mu \mathrm{g} / \mathrm{mL}$ ) and was thus used for subsequent studies. The time taken to reach maximum activation varied among different agonists: 2 min for 2-MeS-ADP; $3 \mathrm{~min}$ for thrombin, thrombin receptor agonist peptide (TRAP; a kind gift from Dr. N. Greco, Holland Laboratory of Red Cross, Rockville, MD, USA), and U46619; and $5 \mathrm{~min}$ for collagen. Figure 1, panels B, C, and E, shows that the collagen activated the platelets in a concentration-dependent manner for all three preparations (WP, PRP, and WB), with the strongest signals detected in WP. Because of the light absorption factor, significantly lower signals were detected in WB. Nevertheless, the activation of platelets by collagen was inhibited in a concentration-dependent manner by milrinone in all three preparations. Milrinone is a potent phosphodiesterase III inhibitor that inhibits platelet aggregation by increasing the intracellular cAMP concentration (Figure 1F). Other inhibitors, such as RGDS (Sigma) and MK383 (Biomol) to inhibit fibrinogen-binding to GPI$\mathrm{Ib} / \mathrm{III}$ a or $\mathrm{PGE}_{1}$ to elevate intra-platelet cAMP levels, were also shown to be inhibitory of collagen-induced ATP release (Figure 1D). Next, we tested the influence of the WP's volume and platelet concentration on signal intensity, following collagen stimulation. The results showed that signal intensity was proportional to the volume added and to the platelet concentration (Figure $2 \mathrm{~A})$. In the range of $1 \times 10^{7}$ to $4 \times 10^{8}$ platelets/mL, the signal detected is linear with the platelet concentration (Figure 2B). ATP was added to test the sensitivity of the assay. A clear signal above the background was detected from $1 \mathrm{pmol} /$ well ATP to $1 \mathrm{nmol} /$ well, in a linear relationship (data not shown). Other agonists could also have been used to stimulate platelet activation in this assay. Figure 3 shows thrombin, TRAP, U46619, and 2-MeSADP concentration-dependently stimulated platelet secretion in WP (for thrombin) and in PRP. Their stimulat- ory effects were countered by PGE1 and by receptor antagonists, such as SQ29548 for thromboxane $\mathrm{A}_{2}$ and A3P5PS for $\mathrm{P}_{2} \mathrm{Y}_{1}$ purinoceptor. Among the tested agonists, thrombin was the strongest, as determined by signal intensity. By using the 96-well format, ADP-induced secretion was only observed in citrated PRP (not in hirudinized PRP), while epinephrin caused no platelet secretion at concentrations up to $100 \mu \mathrm{M}$. When necessary, ATP standards could also be included to quantify the amount of released ATP.

It is possible to induce clotting in the 96-well plate by recalcifying citrate PRP at $37^{\circ} \mathrm{C}$. During the coagulation process,

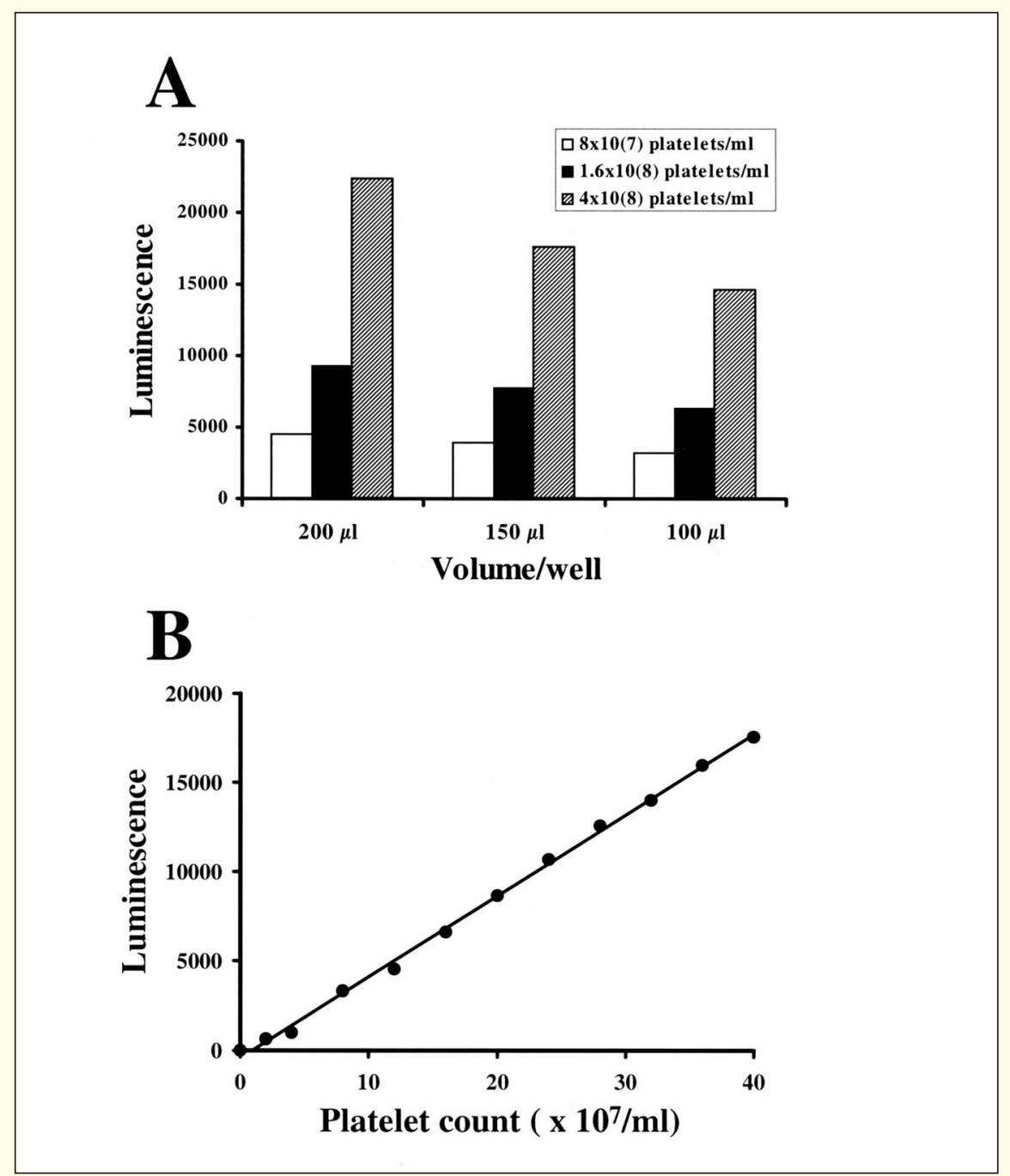

Figure 2. Assessment of assay volume and platelet count on luminescent levels detected by the 96well-based luminometry of platelet activation. (A) Comparison of assay volumes and platelet concentrations of WP on detected signal intensity (arbitrary units). Collagen $(1 \mu \mathrm{g} / \mathrm{mL})$ was used to induce platelet activation. (B) Linear relationship between platelet concentration and luminescence (arbitrary units) detected in WP after stimulation with collagen $(1 \mu \mathrm{g} / \mathrm{mL})$. 


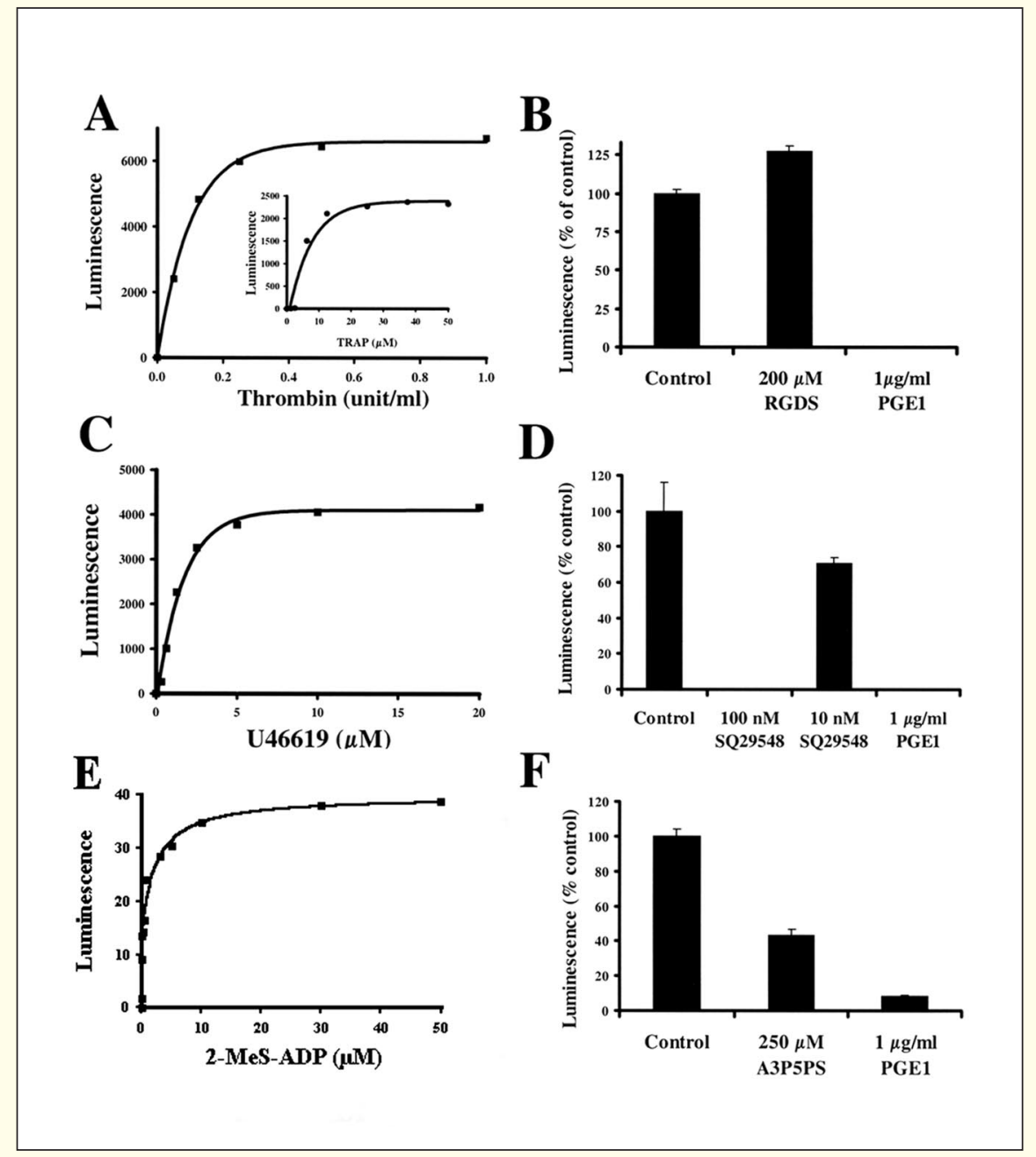

platelets were initially activated by generated thrombin, followed by the secretion of ATP. By detecting released ATP from platelets, the luminometric assay was also suitable for quantifying coagulation. Heparin is a potent coagulation inhibitor that binds to the exosite 2 of thrombin (4). Figure 4 shows that heparin dose-dependently inhibited clotting with an $\mathrm{IC}_{50}$ of about $0.15 \mathrm{U} / \mathrm{mL}$.

Both ATP and ADP are released from platelet-dense granules during activation. ATP is quickly degraded by ecto-ATPase on platelets or in the plasma (6). To prevent this breakdown, we added $500 \mu \mathrm{M}$ creatin phosphate (CP) and $1000 \mathrm{U} / \mathrm{mL}$ creatin phosphokinase (CPK) to the plasma to convert ADP back to ATP. As shown in Figure 5,

Figure 3. Other agonist-induced platelet aggregation as determined by 96-well-based luminometry. Left panel: representatives of dose-dependent effect of thrombin in WP (A) and TRAP (A, insert), U46619 (C), and 2-MeS-ADP (E) in PRP on platelet activation. The platelet concentration in WP for thrombin-induced activation (A) was adjusted to $4 \times 10^{7} / \mathrm{mL}$. Each data point was the mean of duplicate assays. Similar data were obtained from at least two other separate experiments. Luminescence data are in arbitrary units. Right panel: inhibitory effect on thrombin $(0.1$ $\mathrm{U} / \mathrm{mL}$ )-induced platelet activation in WP (B) and U46619 (D) $(2 \mu \mathrm{M})$ or 2-MeS-ADP (F) $(5 \mu \mathrm{M})$ induced platelet activation in PRP; $n=3$. Data are expressed as the percentage of the controls.

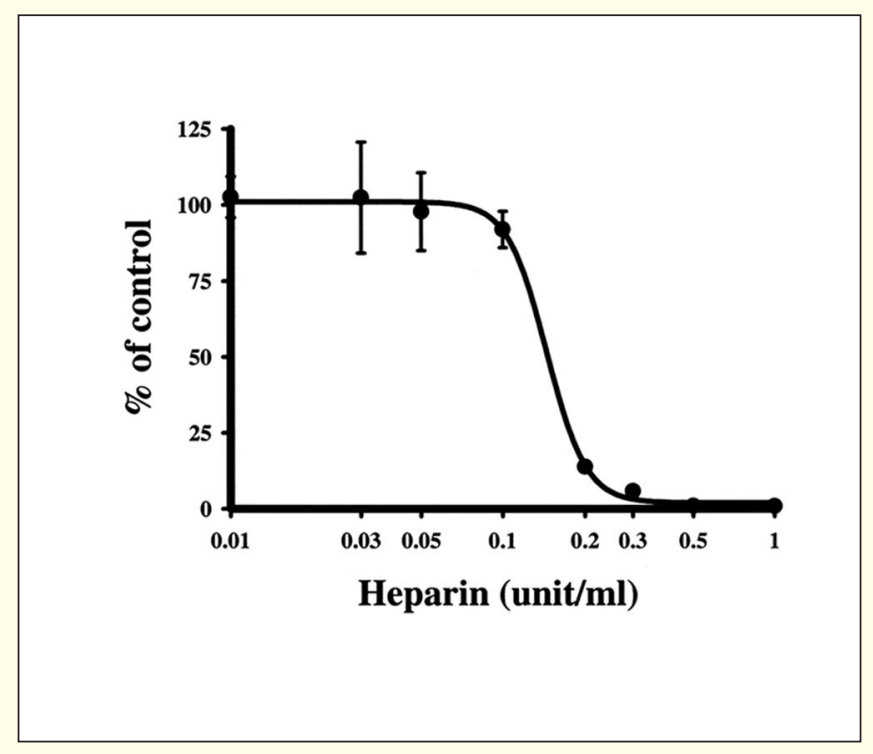

Figure 4. Concentration-dependent inhibition of coagulation of citrate PRP by heparin. Data are the mean of three separate experiments of triplicate assays and are expressed as the percentage of controls (without heparin).

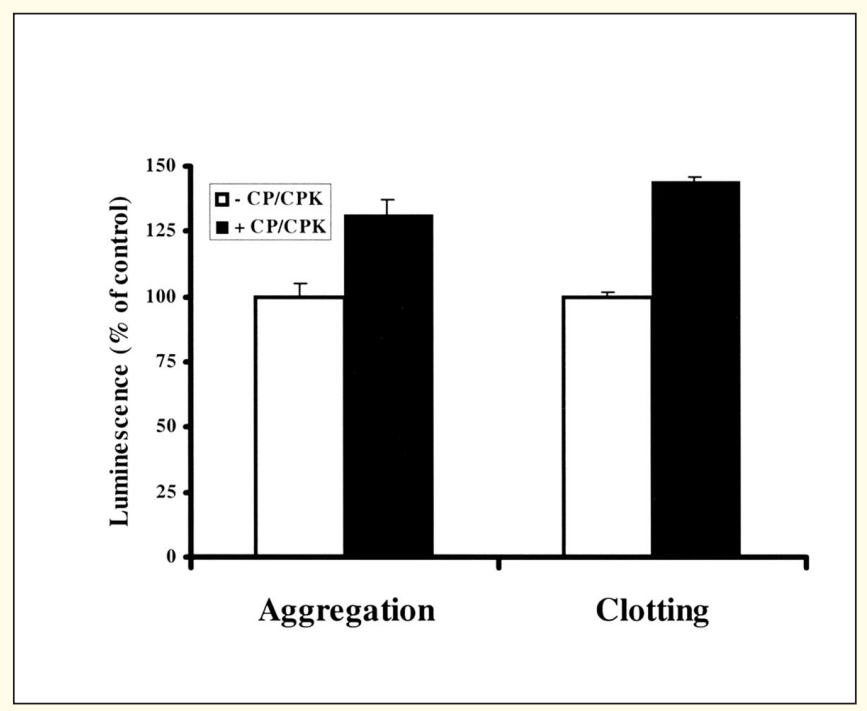

Figure 5. Effects of CP $(500 \mu \mathrm{M})$ and CPK $(1000 \mathrm{U} / \mathrm{mL})$ on collagen $(1$ $\mu \mathrm{g} / \mathrm{mL}$ )-induced platelet aggregation and re-calcification-induced coagulation of citrate PRP $(\boldsymbol{n}=\mathbf{3})$. Data are expressed as the percentage of controls (without CP/CPK). 
$\mathrm{CP} / \mathrm{CPK}$ significantly enhanced the signal level by $30 \%-40 \%$ for both platelet aggregation in PRP and clotting using this assay. In WP, the degradation of ATP is slow, and the signals detected after the addition of substrate were stable for at least $10 \mathrm{~min}$, which is suitable for large-scale screening.

\section{DISCUSSION}

Our studies demonstrated that the 96-well, microplate-based luminometric assay for platelet activation is more sensitive than light transmission with an aggregometer or microplate, as previously reported $(2,5,10,14)$. In these reports, the usual detection range using a microplate was between 0 and 0.3 , with the maximum up to 0.8 OD. Errors can also occur when reading the wells; this is a result of air bubbles introduced into the wells by pipetting. With the luminometric assay, platelet-released ATP can be quantified in a dynamic range of five orders of magnitude. The luminescence detected is not compromised by the presence of air bubbles in the well. In addition, this assay can be used for the detection of platelet activation in WB and for the study of anticoagulants. Because of its high sensitivity, a smaller concentration of platelets (100 times less than with the aggregometer) is required for detecting platelet activation compared to other methods. Therefore, the luminometric assay has the potential to be adopted in other microplate formats such as the 386-well plate. Considering the limited supply of platelets and the short period of time suitable for the study of platelet function following blood collection, this assay is versatile enough for the small research laboratory or for the large-scale screening industry.

Precautions need to be taken into consideration when using this luminometric assay. Some weak agonists such as epinephrin may only cause primary aggregation without degranulation in the microplate. Therefore, this assay is not suitable for detecting epinephrininduced platelet activation. It is also necessary to test the potency of the chosen agonist for each individual blood donor before the screening and to choose the most appropriate stimulating dose, especially for weak agonists such as ADP. Subsequent comprehensive studies with an aggregometer or a flow cytometer are necessary to confirm the initial screening findings.

\section{ACKNOWLEDGMENTS}

The authors thank Mr. Sang N. Le for reviewing the English.

\section{REFERENCES}

1.Baumgartner, H.R. 1973. The role of blood flow in platelet adhesion, fibrin deposition and formation of mural thrombi. Microvasc. Res. 5:167-179.

2.Bednar, B., C. Condra, R.J. Gould, and T.M. Connolly. 1995. Platelet aggregation monitored in a 96 well microplate reader is useful for evaluation of platelet agonists and antagonists. Thromb. Res. 77:453-463.

3.Born, G.V.R. 1962. Aggregation of blood platelets by adenosine diphosphate and its reversal. Nature 194:927-929.

4.Fareed, J., D. Hoppensteadt, J. Fareed, J.M. Walenga, H. Wolf, and R.L. Bick. 1999. Current developments in anticoagulant and antithrombotic agents, p. 495-525. In G.H.R. Rao (Ed.), Handbook of Platelet Physiology and Pharmacology. Kluwer Academic Publishers, Norwell, MA.

5.Fratantoni, J.C. and B.J. Poindexter. 1990. Measuring platelet aggregation with microplate reader. Am. J. Clin. Pathol. 94:613617.

6.Marcus, A., M. Broekman, J. Drosopoulos, D. Pinsky, N. Islam, R. Gayle, III, and C. Maliszewski. 2001.Thromboregulation by endothelial cells: significance for occlusive vascular diseases. Arterioscler. Thromb. Vasc. Biol. 21:178-182.

7.Michelson, A.D., M.R. Barnard, L.A. Krueger, A.L. Frelinger, III, and M.I. Furman. 2000. Evaluation of platelet function by flow cytometry. Methods 21:259-270.

8.O'Brien, J.R. 1962. Platelet aggregation. Part II: some results from a new method of study. J. Clin. Pathol. 15:452-455.

9.Reed, G.L., M.L. Fitzgerald, and J. Polgar. 2000. Molecular mechanisms of platelet exocytosis: insights into the "secrete" life of thrombocytes. Blood 96:3334-3342.

10.Salmon, D.M. 1996. Optimization of platelet aggregometry utilizing microtiter plate technology and integrated software. Thromb. Res. $84: 213-216$

11.Shattil, S.J., M. Cunningham, and J.A. Hoxie. 1987. Detection of activated platelets in whole blood using activation-dependent monoclonal antibodies and flow cytometry. Blood 70:307-315.

12.Sun, B. and J. Kambayashi. 1999. Discrete intracellular $\mathrm{Ca}^{2+}$ pools coupled to two $\mathrm{Ca}^{2+}$ distinct influx pathways in human platelets. J. Biomed. Sci. 7:35-41.

13.Tandon, N.N., U. Kralisz, and G.A.
Jamieson. 1989. Identification of glycoprotein IV (CD36) as a primary receptor for platelet-collagen adhesion. J. Biol. Chem. 264:7576-7583.

14.Walkowiak, B., A. Kesy, and L. Michalec. 1997. Microplate reader-a convenient tool in studies of blood coagulation. Thromb. Res. 87:95-103.

Received 22 May 2001; accepted 10 July 2001.

Address correspondence to:

Dr. Bing Sun

Maryland Research Laboratories

Otsuka Maryland Research Institute, LLC.

9900 Medical Center Drive

Rockville, MD 20850, USA

e-mail: bings@otsuka.com

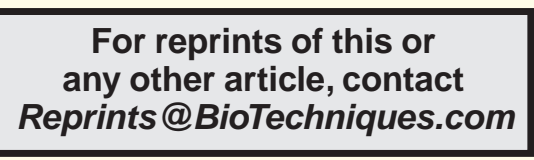

